\title{
(2) OPEN ACCESS \\ Concussion in para sport: the first position statement of the Concussion in Para Sport (CIPS) Group
}

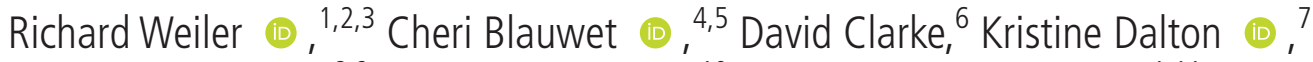 \\ Wayne Derman (1) ${ }^{8,9}$ Kristina Fagher (1) ${ }^{10}$ Vincent Gouttebarge (1) , 1,11

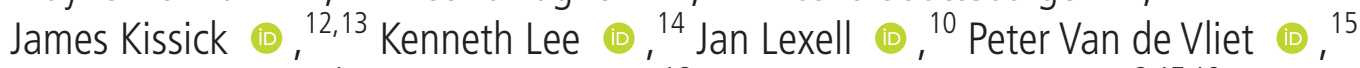 \\ Evert Verhagen (1) , ${ }^{1}$ Nick Webborn (1) , ${ }^{16}$ Osman Hassan Ahmed (1) 3,17,18
}

- Additional supplemental material is published online only. To view, please visit the journal online (http://dx.doi. org/10.1136/bjsports-2020103696)

For numbered affiliations see end of article.

\section{Correspondence to} Professor Evert Verhagen, Department of Public and Occupational Health, Amsterdam UMC, Amsterdam, Noord Holland, The Netherlands; e.verhagen@amsterdamumc.nl

Accepted 4 March 2021 Published Online First 9 April 2021

Check for updates

(c) Author(s) (or their employer(s)) 2021. Re-use permitted under CC BY-NC. No commercial re-use. See rights and permissions. Published by BMJ.

To cite: Weiler $R$,

Blauwet $C$, Clarke D,

et al. Br I Sports Med

2021:55:1187-1195.

\section{ABSTRACT}

Concussion is a frequent injury in many sports and is also common in para athletes. However, there is a paucity of concussion research related to para sport, and prior International Concussion in Sport (CIS) consensus papers have not substantively addressed this population. To remedy this and to improve concussion care provided to para athletes, the concussion in para sport (CIPS) multidisciplinary expert group was created. This group analysed and discussed in-depth para athlete-specific issues within the established key clinical domains of the current (2017) consensus statement on CIS. Due to the onset of the COVID-19 pandemic, the group held all meetings by video conferencing. The existing Sport Concussion Assessment Tool 5 (SCAT5) for the immediate on-field and office-based off-field assessment of concussion was evaluated as part of this process, to identify any para athlete-specific concerns. Regular preparticipation and periodic health examinations are essential to determine a baseline reference point for concussion symptoms but pose additional challenges for the interpreting clinician. Further considerations for concussion management for the para athlete are required within the remove, rest, reconsider and refer consensus statement framework. Considering return to sport (RTS), the 2017 CIS consensus statement has limitations when considering the RTS of the para athlete. Case-by-case decision making related to RTS following concussion is imperative for para athletes. Additional challenges exist for the evaluation and management of concussion in para athletes. There is a need for greater understanding of existing knowledge gaps and attitudes towards concussion among athlete medical staff, coaches and para athletes. Future research should investigate the use and performance of common assessment tools in the para athlete population to better guide their clinical application and inform potential modifications. Concussion prevention strategies and sport-specific rule changes, such as in Para Alpine Skiing and Cerebral Palsy Football, also should be carefully considered to reduce the occurrence of concussion in para athletes.

\section{INTRODUCTION}

Concussion prevention, recognition, assessment and management have been topics of much discussion and debate across all major global sports, including para Sports. Injury surveillance data from the most recent summer and winter paralympic games, showing high rates of injuries to the head and face in football 5 -a-side (blind football), Para
Alpine Skiing, Para Ice Hockey and Para Snowboarding, demonstrate the need for this discussion and debate. ${ }^{12}$ At the Rio 2016 Summer Paralympic Games, no concussions were reported by team physicians in the injury reporting survey, despite several incidents across different sports where athletes were observed to suffer a blow to the head, followed by unsteady gait and reports of significant injuries to the head and face. ${ }^{3}$ Recently, a study of clinicians working at the 2015 Cerebral Palsy (CP) Football World Championships showed that clinicians' overall knowledge around concussion was sound, but their methods for recognition and management were highly variable. ${ }^{4}$ Study participants recognised an urgent need for expert clinical guidance specific to this population of athletes. Aside from a wider need for concussion education, clinicians face difficulties when applying existing concussion assessment and management guidelines to para athletes.

Various terminology has been used within para sport and our paper will align with the Para sport translation of the International Olympic Committee (IOC) consensus on the recording and reporting of data for injury and illness in sport. ${ }^{5}$ Accordingly 'a para athlete is the International Paralympic Committee's term for a sportsperson with an impairment and these terms will be used in this consensus statement as they apply to all athletes with impairment partaking in sport.' The World Health Organization (WHO) defines disability as 'any restriction or lack of ability to perform an activity in the manner or within the range considered normal for a human being,' wherein disability is considered the result of an impairment. ${ }^{5-7}$

While tools assessing brain function (eg, Sport Concussion Assessment Tool 5 (SCAT5) and neurocognitive tests) are widely acknowledged to be helpful following concussion, these tools are neither validated nor applicable in some respects within a para sport population. Significant differences were shown between baseline SCAT3 scores for footballers with and without impairment. ${ }^{8}$ These differences, among others, need to be recognised and considered, in order to create concussion guidelines that are specific to the wide variety of impairments noted in the growing number of athletes living with impairment. Within previous iterations of the consensus statements from the Concussion in Sport Group (CISG), ${ }^{9}$ there has been no consideration of the specific issues faced by para athletes, which may 
reflect the lack of concussion-related research that focuses on para athletes. Despite a paucity of research, the concussion issues faced by para athletes and their support staff are of concern and therefore specific recommendations for standards of care are needed.

\section{METHODS}

The aim of the Concussion in Para Sport (CIPS) group was to develop specific recommendations and guidance related to para athletes and their attending medical professionals in the event that a para athlete sustains a suspected concussion. The Concussion Recognition tool 5 (CRT5), SCAT5 and the CIS 2017 consensus statement lack any specific guidance focusing on the para athlete. Our analysis will focus on the SCAT5, which can be used by physicians and licensed healthcare professionals for athletes aged 13 years or older and encompasses immediate on field assessment and office based off-field assessment of suspected concussion.

An initial multidisciplinary panel of experts was selected who are academically and/or clinically experienced in para sport or concussion in both para and non-para athletes. This group was expanded to include clinicians, researchers, para athletes with experience of concussion and other experts with experience in this field. Initially, a face-to-face meeting was planned for May 2020, however, owing to the COVID-19 pandemic, this was adapted to a video conference split over two consecutive afternoons in June $2020 .^{10}$

The process was conducted over nine phases: (1) initial discussions and selection of members of the consensus group; (2) assignment of designated topic areas to four working groups; (3) review of CISG consensus key sections by each working group, with consideration of issues, key questions and knowledge gaps concerning para athletes; (4) circulation of each working group's written summary of their review and discussions on each designated topic area, (5) a 2-day consensus meeting held on the 15-16 June 2020, during which all contributions were discussed by the whole consensus group section by section; (6) revision of drafts by working groups according to the input received and consensus reached during the meetings; (7) a final consensus meeting held on the 24th of September 2020 to verify and agree on all changes; (8) final edits made by an editorial group (RW, $\mathrm{OHA}$ and EV) and (9) review and approval of the final draft by all consensus group members.

The four working groups were tasked with exploring key clinical areas of CIPS described in the most recent consensus statement of CIS, ${ }^{9}$ and were divided into: concussion assessment; concussion management; return to sport (RTS) following concussion; and specific considerations related to the different impairments in Para athletes.

\section{RECOMMENDATIONS FOR CONCUSSION ASSESSMENT FOR PARA ATHLETES \\ Clinical assessments}

A key point within the SCAT5 highlights that 'the diagnosis of a concussion is a clinical judgement, made by a medical professional'. In light of our considerations of concussion assessment for the para athlete, this becomes even more important. As part of the overall assessment an attending medical professional may choose to seek a corroborative history from suitable family members or carers, if available, to assist them in clinical decision making. Concussion in the para athlete population should, to the extent possible, be managed according to existing concussion consensus guidelines. The SCAT5 should not be used in and of itself to make, or exclude, the diagnosis of concussion in a para athlete, and a para athlete may have a concussion even if their SCAT5 is deemed to be 'normal'. If the clinician has concerns regarding the assessment or return to play (RTP) progression, they should err on the side of caution when making decisions.

In the para athlete population, the importance of clinicians performing routine baseline periodic preparticipation examinations (PPE) is emphasised in order to establish a point of reference for the assessment of concussion symptoms in the future (and also more generally for subsequent injury or illness). This should include consideration of para athletes' baseline cognitive function and their visual or physical abilities. Paradoxically, while SCAT5 baseline testing cannot be mandated for para athletes (due to the lack of validity in general populations and an even greater variability of baseline scores between different disability groups), the clinician attending to a para athlete with a suspected concussion has a much greater need to have a comprehensive understanding of a para athlete's preinjury cognitive function and abilities in order to make a diagnosis of concussion and manage the athlete more effectively. Additionally, it is recommended that a team clinician with prior knowledge of the athlete is involved in the acute assessment of the potentially concussed athlete. The feasibility of this may be limited in some sport contexts, depending on the sport specific rules, (eg, team clinicians may not be permitted access to the field of play) and some teams may not travel with the support of a team physician, physiotherapist or athletic therapist. These shortcomings need to be considered by International Federations, with consideration given to amending regulations for cases in which a head injury has been observed.

\section{Recognise and remove}

In concurrence with the existing SCAT5, any para athlete with a suspected concussion must be removed from practice or play and should not be allowed to return to activity until a medical assessment is conducted (even if their symptoms resolve).$^{11}{ }^{12}$ Emergency medical services must be called immediately if SCAT5 'red flag' signs and symptoms for a severe brain injury are suspected.

Existing concussion recognition and assessment tools (namely the CRT5 ${ }^{11}$ and SCAT5 ${ }^{12}$ ) assume certain 'normal' athlete functions to complete these assessments. They can be used to assess suspected concussion in para athletes, but the clinical user must be aware of limitations. SCAT5 requires an athlete to be able to see, to read, to hear, and to understand with competence, while also having normal baseline speech, language, manual dexterity, and safe range of movement of the cervical spine. These baseline functions may be impaired for some para athletes and it is crucial to recognise this during the initial and following assessments for a suspected concussion. Some para athletes may have a combination of impairments relating to any number of these functions, and these may pose barriers and challenges when trying to undertake head injury assessments following an injury. The validity of any adaptations to concussion assessment tools for para athletes needs to be established.

\section{Para athlete customised assessment tools}

The appendices were collated for each sport specific impairment type using all questions from the immediate or on-field assessment' section of SCAT5 and the 'office or off-field assessment' sections of SCAT5 (online supplemental files). A traffic light system was used, whereby green shading represents no anticipated additional considerations for para athletes, yellow shading represents potential additional considerations for some 
para athletes (dependent on the level or nature of athlete impairment), and red shading represents items from the SCAT5 that should not be used for para athletes. The appendices present specific considerations for the use of each SCAT5 item in the immediate on-field and office or off-field assessments of a suspected concussion in para athletes with:

- (Single) upper limb deficiency.

- (Bilateral) upper limb deficiency.

- (Single) lower limb deficiency.

- (Bilateral) lower limb deficiency.

- Impaired vision.

- Absent vision.

- Globe absent.

- Spinal cord injury (SCI) with quadriplegia.

- SCI with paraplegia.

- CP with spastic diplegia

- CP with spastic hemiplegia.

- CP with spastic quadriplegia.

- Dyskinetic CP.

- Ataxic CP.

- Mixed CP.

- Intellectual impairment.

- Achondroplasia.

- Arthrogryposis.

- Polio.

- Muscular dystrophy.

- Multiple sclerosis.

- Spina bifida.

Examples of SCAT5 sport-specific impairment considerations For immediate assessment, para athletes with total vision loss, regardless of whether the globe is intact, damaged or absent will not be able to report double vision or demonstrate a "blank or vacant' facial expression after a head injury. Furthermore, the best eye response on the Glasgow Coma Scale (GCS) may be limited in non-concussed Para athletes with visual impairments and so a need for a baseline GCS score becomes important to be able to make comparison if they suffer a suspected concussion.

For athletes with Down's syndrome, or achondroplasia or osteogenesis imperfecta, caution is needed when examining the cervical spine because of the associated risk of atlantoaxial instability. Similar caution will be required for those with previous cervical injury as a cause of their existing SCI. Neurological screening tests and use of symptom lists may require alternative methods for communication (eg, Braille or text to speech technology for athletes with visual impairment), or the use of different wording to explain symptoms and visual prompts for athletes with intellectual impairment. For athletes with SCI and other more severe forms of lower limb neurological impairment, use of balance tests is not possible. Alternative tests such as the wheelchair error scoring system (WESS) ${ }^{13}$ if validated in this setting, may emerge as suitable clinical alternatives.

Intellectual impairment (which sometimes is also associated with $\mathrm{CP}$, some neurological disorders, previous significant head injuries, vision impairment and spina bifida) poses significant challenges for the reliable assessment of cognitive function and may affect the results of many SCAT5 cognitive tests. Furthermore, the degree of intellectual impairment may also affect the reliability and repeatability of the assessments as a baseline or comparative tool during recovery. Athletes with arthrogryphosis, post-polio syndrome, muscular dystrophy, multiple sclerosis and spina bifida commonly have challenges with lower limb strength and balance. Results of neurological screening tests are variable depending on the level of impairment, and assessments of these athletes must therefore be interpreted with caution.

\section{CONCUSSION MANAGEMENT FOR PARA ATHLETES}

It is important to be aware of the need for special attention and/ or modification of these recommendations depending on the unique nature of the para athlete's impairment. Table 1 outlines some of the considerations for the management of the Para athlete, based on the 2017 CIS Consensus Statement.

\section{Recognise and remove: once concussion is suspected the athlete should be removed from play or practice}

The most important steps in concussion management are the recognition that a concussion may have occurred, and the subsequent removal of the athlete from the game or practice for further assessment. While there may be challenges relating to the assessment in some cases with the Para athlete, once a concussion is suspected, it is critical that the para athlete is removed to prevent further harm. In some instances, para athletes with certain impairments might require special considerations (eg, para athletes with intellectual impairment may have more difficulty understanding the need for removal from play).

Rest: brief period of physical and cognitive rest (24-48 hours) After concussion there should follow a brief period of 24-48 hours of physical and cognitive rest, during which it is noted that there may be challenges for para athletes who are wheelchair users given their additional energy expenditure needed to propel a wheelchair and engage in wheelchair transfers for daily activities. If these activities lead to an exacerbation of their concussion symptoms, strategies to reduce their exertion may be required, for example, assistance with transfers, and assisting with or reducing the need to self-propel. Athletes with high support needs who typically require some level of assistance for activities of daily living may require 24-hour surveillance to enable them to rest and prevent further injury.

Active rest: gradual and progressive increase in activity while staying below their cognitive and physical exacerbation thresholds (activity should not worsen symptoms)

In most cases, after a few days of rest, athletes should be able to gradually increase their daily activity level as long as their symptoms do not deteriorate. As the para athlete begins to gradually increase their level of physical activity, the mechanism for testing submaximal exercise threshold may require modification. For example, stationary cycling is often used as a submaximal exercise challenge, but this will not be possible for wheelchair users and possibly not for amputees or those with marked leg length discrepancies. Alternative activities such as the use of a hand cycle could be used as an alternative. Balance testing and testing of reaction time may also need to be adapted to accommodate different impairments with specific attention to any differences from baseline.

RTS, reconsider: graduated return to activities, using return to work or school and RTS strategies. Return to work or school should come before RTS

As in the general population, the priority for para athletes attending work or school should be returning to work or school first without a deterioration in symptoms, rather than returning to sport. If applicable in school or work, previously existing accommodations due to disability should remain in place postconcussion to prevent unfamiliar changes to the environment 


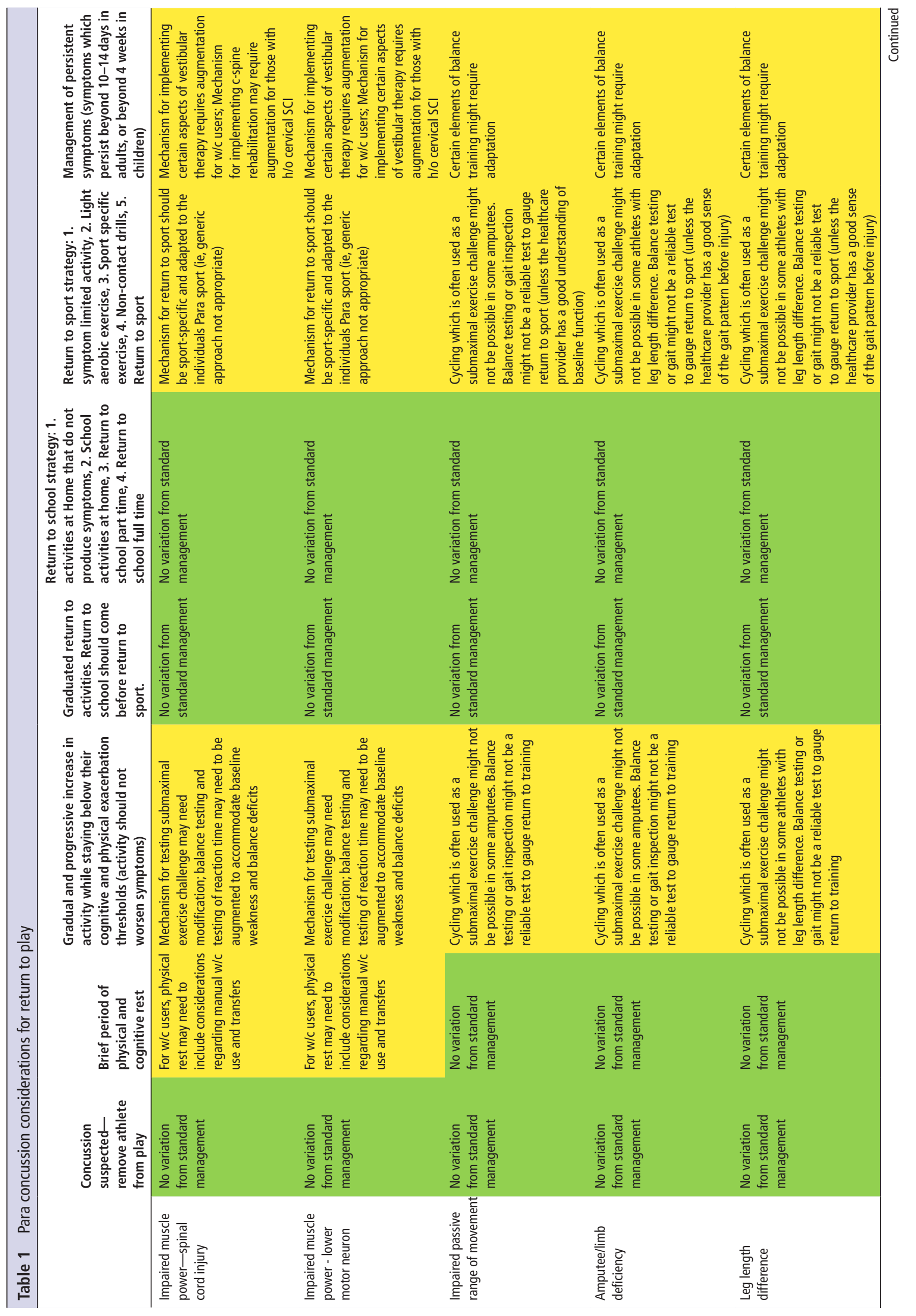

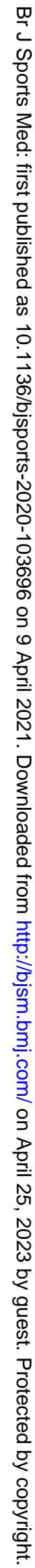




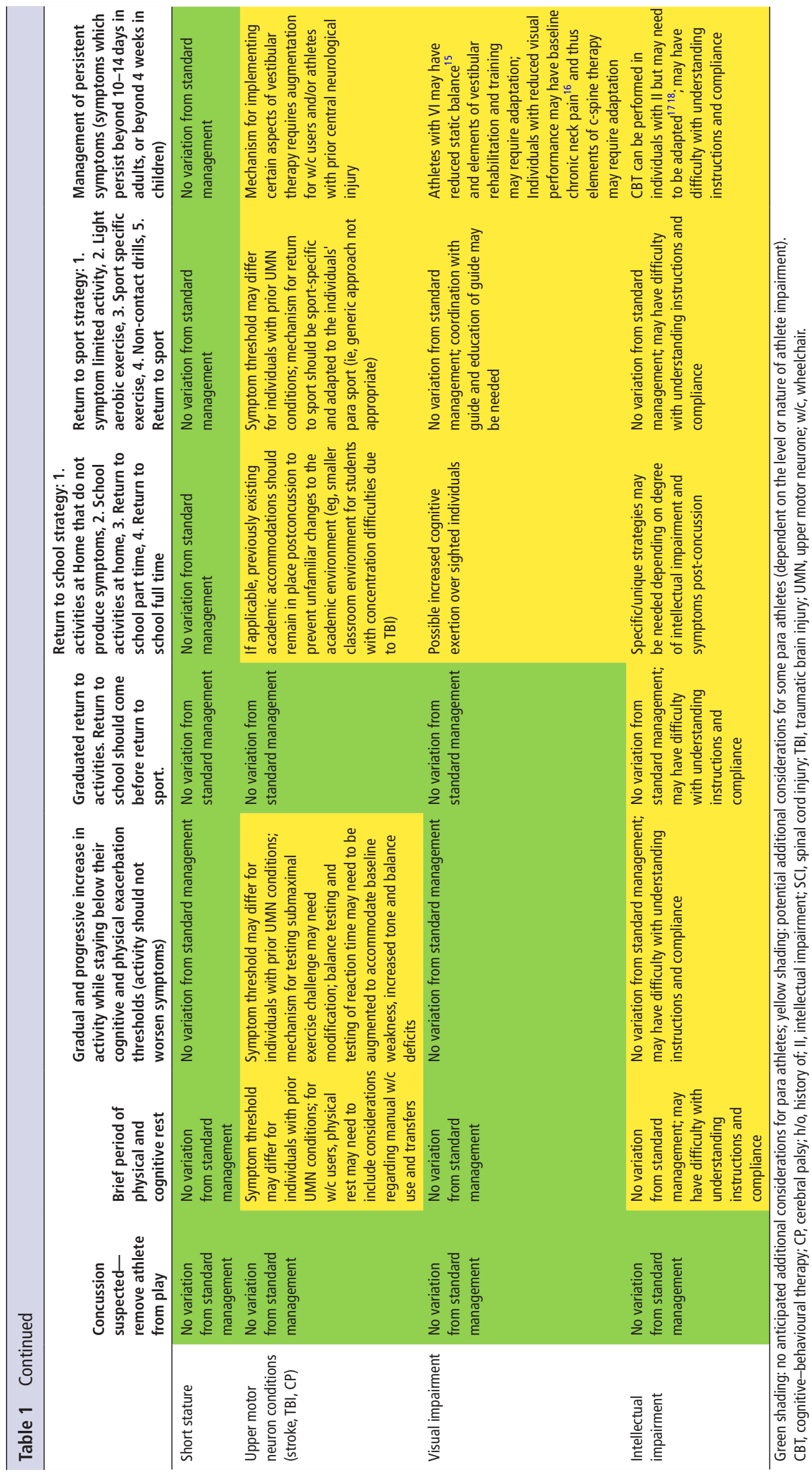


(eg, in school a smaller classroom size for students with concentration difficulties due to intellectual impairment).

As described in the 2017 CIS consensus statement, any RTS strategy should follow a stepwise graduated RTS approach after the initial period of rest and providing they remain free of any symptoms. This should begin with symptom-limited activity, then progression through: light aerobic exercise; sport-specific training; non-contact training drills/resumption of resistance training; contact activity (if applicable); then a RTS. Clinical experience supports that the same strategy be adopted for para athletes, but the mechanism for graduated RTS should be sport specific. For some para athletes, the use of balance and gait testing will not be reliable to help guide their RTS. In the visually impaired athlete competing with a guide, graduated training with the guide is important to simulate the athlete's sport-specific training and competition environment during their gradual return to normal activity. The guide may also be able to observe for signs suggesting recovery has not yet been achieved, for example, abnormal balance.

Refer: management of persistent symptoms (symptoms which persist beyond 10-14 days in adults, or beyond 4 weeks in children)

Some para athletes will have persistent symptoms following a concussion. The 2017 CIS Consensus Statement states that treatment should be individualised to best address specific medical, physical, and psychosocial factors. It reported evidence to support symptom limited aerobic exercise, physiotherapy for cervical spine or vestibular dysfunction, and cognitivebehavioural therapy (CBT) for persistent mood or behavioural issues. ${ }^{14}$ Slight modifications may be needed for the para athlete. For example, the mechanism for implementing certain aspects of vestibular therapy requires augmentation for wheelchair users, athletes with prior central neurologic injury and visually impaired athletes. ${ }^{15}$ The mechanism for implementing cervical spine rehabilitation may require adaptation for those with cervical SCI, and in visually impaired athletes who may report baseline chronic neck pain. ${ }^{16}$ While CBT may be used in para athletes with intellectual impairment, the process by which this occurs may need to be adapted. ${ }^{1718}$

\section{RTS FOLLOWING CONCUSSION IN THE PARA ATHLETE}

The 2017 CIS Consensus Statement ${ }^{9}$ and accompanying SCAT $5^{12}$ outline the current best practice for RTS following concussion, but do not take into account the nuances associated with the para athlete. Furthermore, clinicians and coaches working in para sport have been shown to have gaps in their knowledge relating to RTS following concussion. ${ }^{19}$

In the absence of a current RTS strategy which is specific to the para athlete, the use of the existing RTS strategy from the 2017 CIS Consensus Statement is recommended for para athletes. The RTS strategy recommends an initial 24-48 hours period of physical and cognitive rest before beginning the RTS progression once symptom free. Clinicians are advised to consider whether para athletes may need a longer initial period of physical/cognitive rest prior to starting RTS over and above the 48 hours guideline prior to starting RTS in graduated RTS strategy for impairment specific reasons, for example, if they have a history of previous central nervous system (CNS) injury or impaired CNS function at baseline.

\section{Implications for RTS following concussion in specific para athlete cohorts}

For many para athlete cohorts there may be specific considerations for RTS programmes. While an increasing progression of exertion during RTS is indicated, it should be recognised that para athletes with SCI (T6 and above) may have alterations in their cardiovascular and autonomic responses restricting maximal heart rate (HR), for example. ${ }^{20}$ This would make the use of percentages of maximum HR unreliable, and the use of perceived exertions to monitor the cardiovascular response is advised. As such, a lower level of exertion may be advised for para athletes during this RTS process.

For para athletes with visual impairment, many signs and symptoms that clinicians monitor during the RTS process (eg, dizziness, headache, blurred vision, poor balance) may be preexisting. ${ }^{21}$ Care is needed when distinguishing symptoms elicited during the RTS process from baseline values in this cohort, and it is incumbent on the evaluating clinician to specifically ask about 'change from normal' as opposed to the presence of a symptom. Para athletes with intellectual impairment may have difficulty in understanding the progression and pacing of the different stages in RTS following concussion and are likely to need closer supervision at all stages of the RTS process. Many para athletes (eg, those with $\mathrm{CP}$, stroke, traumatic brain injury) already present with some degree of neurological impairment and a longer/more cautious RTS strategy may be required.

Across all of the para athlete groups, there may be psychological responses to RTS which vary from those of non-para athletes. ${ }^{22}$ Reactions and behaviours related to the process of RTS itself (eg, increased risk taking) may be amplified for para athletes. Since fewer athletes compete in para sport, particularly team sports, extra pressure (intrinsic or extrinsic) may also influence RTS decision making. These reactions and behaviours may be magnified due to the support structures in place around the para athlete, as some coaches working in para sport have been shown to lack knowledge about training and athlete health. ${ }^{23}$

\section{Recommendations for RTS following concussion in the para athlete}

Case-by-case decision making related to RTS postconcussion is crucial for non-para athletes. Given the unique presentations of many para athletes, this individualisation of care is even more paramount. Preparticipation data collected via the SCAT5 (eg, balance and symptoms of headache) and other clinical or athletespecific information may help to provide clinical context when evaluating RTP testing. For athletes who are wheelchair users, walking and running drills in the existing graduated RTS strategy will need to be adjusted to wheelchair-based drills instead. For athletes using wheelchairs who participate in a contact sport (eg, wheelchair rugby, para ice hockey, wheelchair basketball) then full contact practice as part of RTS should be centred around individualised drills, away from other players due to the increased risk of collisions. ${ }^{13}$ Due to the increased risk of concussion in VI sport, ${ }^{2425}$ VI athletes involved in contact sport (eg, blind football, blind judo or goalball) should also conduct their full contact practice as part of RTS away from other players.

Ideally, a para athlete should have the same support during the graduated RTS phases as non-para athletes (eg, a doctor, physiotherapist or athletic therapist knowledgeable in the specific sport that can guide the Para athlete through the different RTS stages). The final decision on when the para athlete returns to sport should always be taken by a doctor with the skills, knowledge and training to make this decision. This is especially pertinent in the instances of para athletes from high-risk populations (eg, those with a preexisting brain injury such as CP, stroke or traumatic brain injury) who have sustained multiple concussions, and where guidance and discussion is needed regarding possible retirement. 


\section{ADDITIONAL CONSIDERATIONS OF CONCUSSION IN PARA ATHLETES}

It is commonly agreed that para athletes can experience the same spectrum of medical conditions as non-para athletes, but there are several considerations that can make the functional consequences of an injury considerably different. A first consideration is the specific context in which the para athlete participates and performs, which differs considerably from the non-para athlete. Often, the supporting structures around para athletes may be less organised and controlled, with reduced access to formalised and organised training. As a result, para athletes often train alone. ${ }^{19}$ There may also be a more limited range of formal competitive sporting events for para athletes, limiting the para athlete's ability to get acquainted with the particular stresses and pressure of competition. ${ }^{26} \mathrm{~A}$ smaller competition programme may offer reduced opportunities for selection and qualification and may add pressure toward early RTS. While it is expected that para athletes practice routine preventive measures to maintain optimal health, it is known that they often do not seek medical consultation for problems considered inherent to their impairment. Consequently, para athletes often try to 'work through' an injury or discomfort and report to medical staff with delay. ${ }^{23} 27$ Additional challenges arise when team staff and medical staff in para sport are appointed on an event base, and during these events there is not always specialised care and support available. This has a natural impact on instances of concussive injury, as the specific context of para athletes and their ability to train and compete demands specific approaches in terms of education and implementation of concussion guidelines.

\section{Prevention}

To develop a successful injury prevention programme, detailed information on the magnitude, burden, risk factors and mechanisms of the health issue are needed. The best available evidence stems from large competitions where valid data are obtained through available event registries. ${ }^{1-3} 2728$ However, the diagnostic accuracy of concussion incidents in these reports remains unknown. Outside of the data from larger competitions, we know little about the actual risk of CIPS, and where prevention efforts should be focused. A risk-rating table has been developed to estimate concussion risk based on sport, impairment, impact speed, collision potential and head protection which provides a starting point. ${ }^{29}$ However, there is further evidence that suggests underreporting or misreporting of CIPS is a significant problem, including a study in wheelchair basketball where $44 \%$ of respondents did not report their concussion. ${ }^{2829}$

The concept of the ' 3 E's of Injury Prevention': education, enforcement and engineering (or environment) are equally applicable to CIPS.

\section{Education}

Education of clinicians, sports technical staff and athletes is crucial. There is an obvious gap in knowledge related to CIPS. This gap must be filled to develop tailored approaches for concussion assessment and management in this population. This applies to generic information about concussion, but more so to para sport specific issues. For example, while concussion in the Para athlete may impact sports participation, there is also an impact on the para athlete's ability to participate in daily life and potentially other disability-related consequences. Given the lesser availability of specially trained personnel and medical staff for para athletes during training and competition, there may also be an onus on the Para athlete to recognise and report concussive symptoms. The implementation of concussion guidelines in para sport should, therefore, include the notion that the application of these guidelines should be possible for non-medically trained users (eg, coaches). In turn, placing further demands on concussion education and training of the appropriate end-users in para sport that is pitched at an appropriate level.

The dissemination of guidelines for RTS following CIPS should be tailored towards an athlete-friendly format that is accessible to the needs of the specific athlete (eg, using a speech synthesiser, braille, assistive writing technology or large font). In para sport some coaches may be former para athletes themselves, and if they are overseeing part of the graduated RTS process then it is important to consider accessibility for any RTS information also provided to them. Para athletes with intellectual impairment will require guidelines that are easy-to-read and will need sufficient support with comprehending and following these guidelines.

\section{Enforcement}

Enforcement relates to the rules or laws of the sport and changes can be made to enhance safety. As an example, a critical analysis of the high number and nature of injuries during the Para Alpine Skiing during the 2014 Paralympic Winter Games in Sochi ${ }^{30}$ led to a closer collaboration and interaction between medical and sport-technical experts, resulting in an action plan that was successfully implemented in the subsequent 2018 Paralympic Winter Games in PyeongChang. These measures included: adjustments to the slope settings; more training runs; and real-time interaction between the medical team and the sport officials during races. ${ }^{31}$ Such examples may serve as illustrations for other sports to realise supportive changes in policy and, more importantly, in rules. Most recently, World Para Alpine Skiing, World Para Snowboard and World Para Nordic Skiing have inserted concussion specific rules in the sport-technical rules. These allow for non-medically trained individuals (eg, a technical delegate) to initiate a primary concussion assessment in accordance with the FIS Alpine Skiing protocols. Similarly, CP Football has led by example through introducing a concussion substitution rule. ${ }^{32} 33$ In case of a suspected concussion, there is a $10 \mathrm{~min}$ window in which a substitute player may enter the field while the injured player is being assessed for concussion. If the player can continue play, he or she can return to the field without the loss of a substituted player. If he or she needs to forfeit the game, the substituted player is already on the pitch. This allows the medical staff to carefully assess and decide on the suspected concussion without the pressure of an athlete number disadvantage or time.

\section{Engineering (for environment)}

The role of protective equipment in concussion prevention is a subject of much debate and research in some professional sports. Its role has yet to be fully evaluated in para sports, however there is early work by the International Blind Sports Association (IBSA) for the sport of Football 5-a-side. In Football 5-a-side (also commonly known as blind football) athletes are blindfolded on the field of play to ensure athletes with some vision are not advantaged and players orient themselves to the sound of a ball that has integrated bells. Head-to-head contact cannot always be avoided and given anecdotal evidence of concussion, IBSA initiated an internal study on the feasibility of softshell helmets with integrated goggles. There is not enough known to recommend protective equipment currently in prevention of concussion. 
Crucial to all considerations is a continued dialogue between medical experts, sport technical experts and athletes, so that all parties have their input to the proposed measures and targeted outcomes, assessment of feasibility and ultimately acceptance of any proposed policy or ruling.

\section{SUMMARY}

Clinicians working with para athletes must fully understand and appreciate the limitations when applying existing concussion consensus guidance to the para athlete population. 'Normal' baseline functions cannot be assumed in para sport and while validity of any adaptations to assessment tools needs to be established, it is essential that clinicians working with para athletes are familiar with an athletes' baseline or uninjured cognitive and neuromuscular function. This familiarity can only be established through regular periodic PPEs and consideration of the use of SCAT5 and other neurocognitive assessment tools.

In the meantime, concussion in the para athlete population should, to the extent possible, be managed according to existing concussion consensus guidelines. If the clinician has concerns regarding the assessment or return to play progression, they should err on the side of caution when making decisions. The quotation from the 2017 CIS Consensus Statement that 'diagnosis of a concussion remains a clinical judgement, made by a medical professional' is potentially even more relevant for Para athletes. The SCAT5 should not be used by itself to make or exclude the diagnosis of concussion in a para athlete, and a para athlete may have a concussion even if their SCAT5 is deemed to be 'normal'. All aspects of our first position statement as the CIPS group should be considered with the immediate and postconcussion assessments, management, RTS and additional considerations when providing care for a para athlete with a suspected concussion.

\section{What is already known?}

- Injury surveillance data from the most recent summer and winter Paralympic Games, demonstrates high rates of injuries to the head and face.

- Despite a paucity of research, the concussion issues faced by para athletes and their support staff are of concern and therefore specific recommendations for standards of care are needed.

- Clinical tools assessing brain function are widely acknowledged to be helpful following concussion, but these tools are neither validated nor applicable in many respects within a para sport population.

\section{What are new findings?}

- Medical professionals should continue to use Sport Concussion Assessment Tool 5 (SCAT5) for 'on' and 'off' field concussion assessment for para athletes and the Concussion in Para Sport appendices can serve as a guide to interpret these results.

- Para athletes may have a concussion even if their SCAT5 is deemed to be 'normal'. Consequently, SCAT5 should not be used by itself to diagnose concussion in para athletes.

- Given the challenges of diagnosing concussion in para athletes, periodic baseline pre participation evaluations are essential as a point of reference.
Research relating to all aspects of concussion in para athletes is urgently needed to develop a better understanding of the intricacies of this injury in this population. This research will facilitate the process of generating a consensus statement on CIPS, which in turn will improve the safety and welfare of all athletes and staff. In the meantime, regular periodic examinations for para athletes are needed as a reference point post-concussion, in association with improved concussion education and awareness for para clinicians, coaches and athletes. The CIPS will work collaboratively with major sporting organisations to help achieve these aims.

\section{Author affiliations}

${ }^{1}$ Amsterdam Collaboration on Health and Safety in Sports, Department of Public and Occupational Health, EMGO Institute for Health and Care Research, VU University Medical Centre Amsterdam, Amsterdam, Noord-Holland, The Netherlands

${ }^{2}$ Sport \& Exercise Medicine, Fortius Clinic, London, UK

${ }^{3}$ Para Football Foundation, Arnhem, The Netherlands

${ }^{4}$ Department of Physical Medicine and Rehabilitation, Spaulding Rehabilitation; Spaulding Hospital/Brigham and Women's Hospital, Harvard Medical School, Boston, Massachusetts, USA

${ }^{5}$ Kelley Adaptive Sports Research Institute, Boston, Massachusetts, USA

${ }^{6}$ University of Hertfordshire, Hatfield, UK

${ }^{7}$ School of Optometry \& Vision Science, University of Waterloo, Waterloo, Ontario, Canada

${ }^{8}$ Institute of Sport and Exercise Medicine, Division of Orthopaedic Surgery, Department of Surgical Sciences, Stellenbosch University Faculty of Medicine and Health Sciences, Cape Town, South Africa

${ }^{9}$ IOC Research Center, South Africa, South Africa

${ }^{10}$ Rehabilitation Medicine Research Group, Department of Health Sciences, Lund University, Lund, Sweden

11Department of Orthopaedic Surgery, Amsterdam UMC, University of Amsterdam, Amsterdam Movement Sciences, Amsterdam, The Netherlands

${ }^{2}$ Department of Family Medicine, University of Ottawa, Ottawa, Ontario, Canada ${ }^{13}$ International Paralympic Committee Medical Committee, Ottawa, Ontario, Canada ${ }^{14}$ Spinal Cord Injury/Disorder, Physical Medicine \& Rehabilitation, Medical College of Wisconsin, Milwaukee, Wisconsin, USA

${ }^{15}$ International Paralympic Committee, Bonn, Germany

${ }^{16} \mathrm{Centre}$ for Sport and Exercise Science and Medicine, University of Brighton, Eastbourne, UK

${ }^{17}$ University Hospitals Dorset NHS Foundation Trust, Poole, UK

${ }^{18}$ School of Sport, Health and Exercise Science, University of Portsmouth, Portsmouth, UK

Twitter Cheri Blauwet @CheriBlauwetMD, David Clarke @ClarkieGB7, Wayne Derman @WDerman, Kristina Fagher @KristinaFagher, Vincent Gouttebarge @Vgouttebarge, James Kissick @Jay_Doc4, Jan Lexell @JanLexell, Evert Verhagen @Evertverhagen, Nick Webborn @SportswiseUK and Osman Hassan Ahmed @osmanhahmed

Contributors An initial multidisciplinary panel of experts group was selected, who are academically and/or clinically experienced in para sport or concussion. This group was expanded to include clinicians, researchers and other experts with experience in this field. Initially, a face-to-face meeting was planned for May 2020, however, owing to the COVID-19 pandemic, this was adapted to a video conference split over 2 consecutive afternoons in June 2020. The process was conducted over 9 phases: (1) initial discussions and selection of members of the consensus group; (2) assignment of designated topic areas to four working groups; (3) review of CISG consensus key sections by each working group, with consideration of issues, key questions and knowledge gaps concerning para athletes; (4) circulation of each working group's written summary of their review and discussions on each designated topic area, (5) a 2-day consensus meeting held on the 15-16 June 2020, during which all contributions were discussed by the whole consensus group section by section; (6) revision of drafts by working groups according to the input received and consensus reached during the meetings; (7) a final consensus meeting held on the 24 September 2020 to verify and agree all changes; (8) final edits made by an editorial group (RW, OHA and EV); (9) review and approval of the final draft by all consensus group members. The four working groups were tasked with exploring key clinical areas of concussion in para sport described in the most recent consensus statement of concussion in sport, and were divided into: concussion assessment; concussion management; return-to-sport following concussion; and specific considerations related to the different impairments in Para athletes.

Funding The authors have not declared a specific grant for this research from any funding agency in the public, commercial or not-for-profit sectors.

Competing interests None declared. 
Patient consent for publication Not required.

Provenance and peer review Not commissioned; externally peer reviewed.

Supplemental material This content has been supplied by the author(s). It has not been vetted by BMJ Publishing Group Limited (BMJ) and may not have been peer-reviewed. Any opinions or recommendations discussed are solely those of the author(s) and are not endorsed by BMJ. BMJ disclaims all liability and responsibility arising from any reliance placed on the content. Where the content includes any translated material, BMJ does not warrant the accuracy and reliability of the translations (including but not limited to local regulations, clinical guidelines, terminology, drug names and drug dosages), and is not responsible for any error and/or omissions arising from translation and adaptation or otherwise.

Open access This is an open access article distributed in accordance with the Creative Commons Attribution Non Commercial (CC BY-NC 4.0) license, which permits others to distribute, remix, adapt, build upon this work non-commercially, and license their derivative works on different terms, provided the original work is properly cited, appropriate credit is given, any changes made indicated, and the use is non-commercial. See: http://creativecommons.org/licenses/by-nc/4.0/.

\section{ORCID iDs}

Richard Weiler http://orcid.org/0000-0002-6216-839X

Cheri Blauwet http://orcid.org/0000-0001-8568-1009

Kristine Dalton http://orcid.org/0000-0002-2616-4797

Wayne Derman http://orcid.org/0000-0002-8879-177X

Kristina Fagher http://orcid.org/0000-0002-9524-7553

Vincent Gouttebarge http://orcid.org/0000-0002-0126-4177

James Kissick http://orcid.org/0000-0002-7748-9225

Kenneth Lee http://orcid.org/0000-0002-9378-673X

Jan Lexell http://orcid.org/0000-0001-5294-3332

Peter Van de Vliet http://orcid.org/0000-0002-1434-3659

Evert Verhagen http://orcid.org/0000-0001-9227-8234

Nick Webborn http://orcid.org/0000-0003-3636-5557

Osman Hassan Ahmed http://orcid.org/0000-0002-1439-0076

\section{REFERENCES}

1 Derman W, Runciman P, Jordaan E, et al. High incidence of injuries at the Pyeongchang 2018 Paralympic winter games: a prospective cohort study of 6804 athlete days. Br J Sports Med 2020;54:38-43.

2 Webborn N, Cushman D, Blauwet CA, et al. The Epidemiology of Injuries in Football at the London 2012 Paralympic Games. PM\&R 2016;8:545-52.

3 Derman W, Runciman P, Schwellnus $M$, et al. High precompetition injury rate dominates the injury profile at the Rio 2016 Summer Paralympic Games: a prospective cohort study of 51198 athlete days. Br J Sports Med 2018;52:24-31.

4 Griffin S, West LR, Ahmed OH, et al. Concussion knowledge, attitudes, and beliefs amongst sports medicine personnel at the 2015 Cerebral Palsy Football World CHAMPIONSHIPS. Br J Sports Med 2017:51:325

5 Derman W, Badenhorst M, Blauwet C, et al. Para sport translation of the IOC consensus on recording and reporting of data for injury and illness in sport. Br J Sports Med 2021;55:1068-76.

6 World Health Organization. Towards a common language for Functioning, Disability and Health - ICF, 2002. Available: https://www.who.int/classifications/icf/ icfbeginnersguide.pdf [Accessed 8 Jan 2021].

7 World Health Organization. The International classification of functioning. disability and health, 2001. Geneva. Available: https://apps.who.int/iris/bitstream/handle/ 10665/42407/9241545429.pdf [Accessed 28-03-2021].

8 Weiler R, van Mechelen W, Fuller C, et al. Do neurocognitive SCAT3 baseline test scores differ between footballers (soccer) living with and without disability? A crosssectional study. Clin J Sport Med 2018;28:43-50.

9 McCrory P, Meeuwisse W, Dvořák J, et al. Consensus statement on concussion in sport-the $5^{\text {th }}$ international conference on concussion in sport held in Berlin, October 2016. Br J Sports Med 2017;51:838-47.
10 Ahmed $\mathrm{OH}$, Verhagen E, Derman W. International sports and exercise medicine consensus meetings in the COVID-19 era: Zoom-ing across borders. BJSM Blog, 2020. Available: https://blogs.bmj.com/bjsm/2020/07/31/international-sports-andexercise-medicine-consensus-meetings-in-the-covid-19-era-zoom-ing-across-borders/ [Accessed 28-03-2021].

11 Echemendia RJ, Meeuwisse W, McCrory $\mathrm{P}$, et al. The concussion recognition tool 5th edition (CRT5): background and rationale. Br J Sports Med 2017;51:870-1.

12 Sport concussion assessment tool - 5th edition. Br J Sports Med 2017:51:851-8.

13 Wessels K. Concussion assessment in wheelchair users: quantifying seated postural control. University of Illinois at Urbana-Champaign, 2013. Available: https://www. ideals.illinois.edu/bitstream/handle/2142/46829/Karla_Wessels.pdf? sequence $=1 \&$ isAllowed=y [Accessed 8 Jan 2021].

14 Manley G, Gardner AJ, Schneider KJ, et al. A systematic review of potential long-term effects of sport-related concussion. Br J Sports Med 2017;51:969-77.

15 da Silva ES, Fischer G, da Rosa RG, et al. Gait and functionality of individuals with visual impairment who participate in sports. Gait Posture 2018;62:355-8.

16 Sánchez-González MC, Gutiérrez-Sánchez E, Sánchez-González J-M, et al. Visual system disorders and musculoskeletal neck complaints: a systematic review and metaanalysis. Ann N Y Acad Sci 2019;1457:26-40.

17 Roberts L, Kwan S. Putting the C into CBT: cognitive challenging with adults with mild to moderate intellectual disabilities and anxiety disorders. Clin Psychol Psychother 2018;25:662-71

18 Hronis A, Roberts L, Kneebone II. A review of cognitive impairments in children with intellectual disabilities: implications for cognitive behaviour therapy. Br J Clin Psychol 2017;56:189-207.

19 West LR, Griffin S, Weiler R, et al. Management of concussion in disability sport: a different ball game? Br J Sports Med 2017;51:1050-1.

20 West CR, Wong SC, Krassioukov AV. Autonomic cardiovascular control in Paralympic athletes with spinal cord injury. Med Sci Sports Exerc 2014;46:60-8.

21 Fagher K. 118 S. sports-related injuries and illnesses in Paralympic athletes. Lund: Lund University: Faculty of Medicine. Doctoral Thesis, 2019. https://portal.research.lu. se/portal/en/publications/sportsrelate d-injuries-and-illnesses-in-paralympic-athletes( ac2429cd-eee1-4747-8e93-b1475b1d69b1).html

22 Ahmed OH, Slater M, Barker JB. Concussion in athletes with disabilities. In: Bloom GA, Caron JG, eds. Psychological aspects of sport-related concussions, 2019.

23 Fagher K, Forsberg A, Jacobsson J, et al. Paralympic athletes' perceptions of their experiences of sports-related injuries, risk factors and preventive possibilities. Eur J Sport Sci 2016;16:1240-9.

24 Fagher K, Dahlström Örjan, Jacobsson J. Prevalence of Sports-Related injuries and illnesses in Paralympic athletes. PM\&R 2020;12:271-80.

25 Fagher K, Hassan Ahmed 0, Pernheim N, et al. Prevalence of sports-related injuries in Paralympic judo: an exploratory study. J Sci Med Sport 2019;22:902-6.

26 Van de Vliet P. Let's talk facts - What healthcare providers really need to know about Paralympic athletes. Aspetar Sports Med J 2016;5:74-9.

27 Derman W, Schwellnus M, Jordaan E. Clinical characteristics of 385 illnesses of athletes with impairment reported on the WEB-IISS system during the London 2012 Paralympic Games. PM\&R 2014;6:S23-30.

28 Wessels KK, Broglio SP, Sosnoff JJ. Concussions in wheelchair basketball. Arch Phys Med Rehabil 2012;93:275-8.

29 Kissick J, Webborn N. Concussion in para sport. Phys Med Rehabil Clin N Am 2018;29:299-311.

30 Derman W, Blauwet C, Webborn N, et al. Mitigating risk of injury in alpine skiing in the Pyeongchang 2018 Paralympic winter games: the time is now! Br J Sports Med 2018;52:419-20.

31 Blauwet C, Webborn N, Kissick J, et al. When van Mechelen's sequence of injury prevention model requires pragmatic and accelerated action: the case of para alpine skiing in Pyeong Chang 2018. Br J Sports Med 2019:53:1390-1.

32 IFCPF. IFCPF unveils new temporary concussion substitution policy, 2020. Available: https://www.ifcpf.com/news/ifcpf-unveils-new-temporary-concussion-substitution-\% 28tcs\%29-policy [Accessed 8 Jan 2021].

33 Ahmed OH, Fulcher M, Malone D. The introduction of temporary concussion substitutions in disability football: Are we 'headed' in the right direction? Football Med Perform 2020;32:13-17. 